\title{
A Time and Frequency Synchronization Scheme for Multiuser OFDM
}

\author{
Jan-Jaap van de Beek, Per Ola Börjesson, Member, IEEE, Marie-Laure Boucheret, Member, IEEE, \\ Daniel Landström, Julia Martinez Arenas, Per Ödling, Associate Member, IEEE, \\ Christer Östberg, Mattias Wahlqvist, and Sarah Kate Wilson
}

\begin{abstract}
We present a multiuser synchronization scheme for tracking the mobile's uplink time and frequency offsets. It uses the redundancy introduced by the cyclic prefix and does not need additional pilots. We show performance results of an orthogonal frequency division multiplexing (OFDM)-based radio interface based on universal mobile telecommunication system (UMTS) parameters. For a UMTS-typical mobile channel environment, the performance of a coherent system employing the scheme is virtually indistinguishable from the performance of a perfectly synchronized system. In a differentially modulated system, synchronization errors decrease the system performance by about $0.7 \mathrm{~dB}$ compared to a perfectly synchronized system.
\end{abstract}

Index Terms - Communication system, delay estimation, frequency estimation, multicarrier, multiuser system, orthogonal frequency division multiplexing (OFDM), synchronization, universal mobile telecommunication system (UMTS).

\section{INTRODUCTION}

$\mathbf{O}$ RTHOGONAL frequency division multiplexing (OFDM) has been proposed for multiuser systems such as the universal mobile telecommunication system (UMTS) [1] and wireless local area networks (WLAN's) [2]. In multiuser OFDM, the orthogonality of the subcarriers facilitates a subcarrier division of different users, where one OFDM symbol contains many users. In the uplink of such systems, users must be aligned in time and frequency to maintain the orthogonality of the subcarriers. How to synchronize different users in the uplink of such a multiuser OFDM-based system has been unclear so far and is a frequently raised question.

Multiuser OFDM uplink synchronization is more difficult than synchronization in a broadcast or downlink scenario for a couple of reasons. First, because one OFDM symbol carries data for many users, the correction of one user's

Manuscript received September 1, 1998; revised July 25, 1999. This paper was presented in part at the 48th IEEE Vehicular Technology Conference VTC'98, May 1998, Ottawa, Ontario, Canada.

J.-J. van de Beek is with Nokia Svenska AB, Stockholm SE-164 25 Sweden

P. O. Börjesson, D. Landström, and P. Ödling are with the Department of Applied Electronics, Lund University, Lund SE-221 00 Sweden.

M.-L. Boucheret is with the École Nationale Supérieure des Télécommunications, Toulouse 4004 CEDEX France.

J. Martinez Arenas and M. Wahlqvist are with Ericsson Radio Systems AB, Stockholm SE-164 80 Sweden.

C. Östberg is with Ericsson Mobile Communications AB, Lund SE-221 83 Sweden.

S. K. Wilson is with the Division of Signal Processing, Luleå University of Technology, Luleå SE-971 87 Sweden.

Publisher Item Identifier S 0733-8716(99)08957-X. time and frequency offsets cannot be accomplished at the base station receiver, as the correction to one user would misalign other initially aligned users. In our scheme, we have the base station estimate time and frequency offsets, then transmit the information to the mobile. The mobile can then adjust its transmitted signal so that it is in alignment with the other users' signals. This method of having the base station feedback timing information is also used in time division multiple access (TDMA) systems, such as the global system for mobile communication (GSM) [3]. Second, estimation of time and frequency offsets is more difficult in such a multiuser system. The performance of the estimator in [4], for example, decreases as the number of subcarriers assigned to each user decreases. The receiver signal-to-noise ratios (SNR's) also vary for different users. Proper synchronization is, however, necessary to keep the orthogonality of the users, which is essential for reliable transmission. This paper describes a novel synchronization scheme for tracking in the uplink of multiuser OFDM systems. Acquisition of the symbol clock and carrier frequency is discussed in [5]. Our scheme is generally applicable to OFDM-based systems where users are separated in bands of adjacent subcarriers. The work presented in this paper was motivated by the UMTS proposal [1]. We evaluate the scheme for a target system similar to this proposal.

Symbol time and carrier frequency offset estimation methods for OFDM transmission systems have been presented in a number of contributions, see, e.g., [4], [6], and [7]. Most of these offset estimators are explicitly evaluated for a broadcast or downlink scenario. Multiuser synchronization provides some extra challenges not addressed in these papers. Our synchronization scheme contains an implementable time and frequency offset estimator structure based on results in [4]. This estimator uses the redundancy in the received signal due to the cyclic prefix. We adapt this estimator to properties of the multiuser system and the fading channel. The estimates of the users' offsets are returned on a downlink control channel to the mobile transmitters, which adapt their clocks and oscillators to the free-running reference clocks and oscillators at the base station.

Whereas frequency requirements on the estimator performance are tight (see, e.g., [8]), the time offset requirements are relaxed by an additional extension of the cyclic prefix. If a time offset error is within the range of this extension, a channel estimation algorithm, required for coherent detection, acts as a fine-tuning time synchronizer. In differential systems, 
which do not employ channel estimation and equalization, this extra extension provides some robustness too. The absence of a channel equalizer with its fine-tuning synchronizing capabilities in these systems, however, causes a small performance degradation compared to coherent systems. We show by simulation that the time and frequency offset estimator satisfies both the tight frequency requirements and the coarse time requirements.

This paper is organized as follows. We first describe the multiuser OFDM scenario in Section II. In particular, we focus on the multiple access schemes OFDM offers. In Section III, we focus on synchronization of a multiple access scheme where users are divided across the subcarriers and potentially also in time. We discuss the system sensitivity to time and frequency offsets and present the novel synchronization scheme including the offset estimator at the base station. In Section IV, we then present simulation results illustrating the performance of the offset estimation algorithm and the system performance in terms of symbol error rate, and we conclude this paper with Section $\mathrm{V}$, which summarizes the main results.

\section{Multiuser OFDM}

\section{A. OFDM Transmission and Multiple Access}

In an OFDM transmission system, the available spectrum is accessed by a large number of subcarriers. Data symbols are efficiently modulated on these carriers by means of a fast Fourier transform (FFT) [9], both in the uplink and the downlink. We assume a frequency division duplex scheme and concentrate on the uplink frequency band. In a multiuser mobile environment, an OFDM scheme has two main advantages. First, the receiver does not require an adaptive timedomain equalizer if a cyclic prefix is properly used and if the channel does not change much during one OFDM symbol [10]. Secondly, dynamic channel assignment across the spectrum is straightforward as each user can conveniently access all of the subcarriers by the FFT-implemented modulation.

Fig. 1 illustrates the OFDM transmission technique. The complex data symbols $x_{n}$ are coherently modulated on $N$ subcarriers by an inverse discrete Fourier transform (IDFT), and the last $L$ samples are copied and put as a preamble (cyclic prefix) to form the OFDM symbol. This data vector is serially transmitted over a discrete-time channel, whose impulse response is shorter than $L$ samples. At the receiver, the cyclic prefix is removed, and the signal $r_{k}$ is demodulated with a discrete Fourier transform (DFT). In OFDM systems employing this cyclic prefix, the frequency-selective channel distortion appears as a multiplicative distortion of the transmitted data symbols [9], and the received data symbol during the $p$ th OFDM symbol at the $n$th subcarrier becomes

$$
\begin{array}{rl}
y_{n, p}=h_{n, p} x_{n, p}+n_{n, p} & n=0, \ldots, N-1, \\
& p=-\infty, \ldots, \infty
\end{array}
$$

where $h_{n, p}$ is the channel attenuation at the $n$th subcarrier during the $p$ th OFDM symbol and $n_{n, p}$ is additive white Gaussian noise (AWGN).

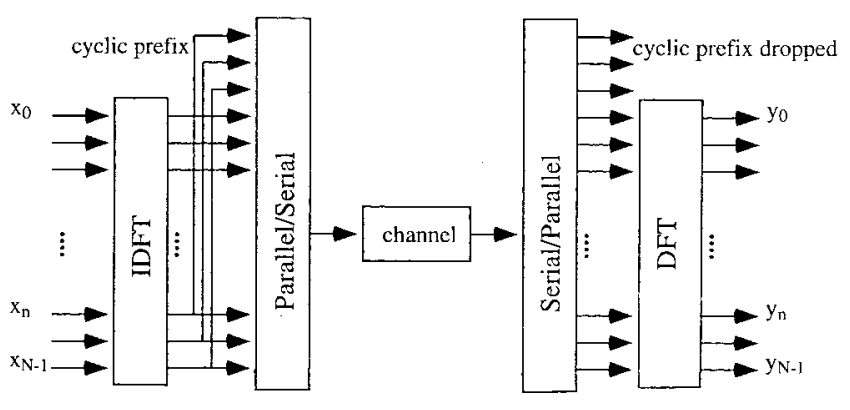

Fig. 1. An OFDM system.

Data symbols can modulate the subcarriers coherently or differentially. Proposal [1] suggests a differential scheme across the subcarriers in an OFDM symbol (a coherent extension is suggested for future system extensions). In Section IV, we evaluate our synchronization scheme for both a differential and a coherent modulation scheme. For coherent modulation, the base station receiver must estimate and compensate for the channel attenuations. For this purpose, pilot symbols are transmitted. Our simulations confirm that channel equalization can act as a fine-tuning synchronizer [11], [12], making coherent systems less sensitive to synchronization errors than differential systems.

Because OFDM separates symbols in both time and frequency, it allows for a number of multiple access schemes. First, in a TDMA structure, users are assigned entire OFDM symbols, and they share the channel by a time-slot structure. Secondly, the large number of subcarriers allows for a frequency division multiple access (FDMA)-like multiple access scheme, where different users are assigned different subcarriers. Since OFDM subcarriers spectrally overlap, this scheme is not true FDMA. However, for convenience, we will use the acronym FDMA for OFDM-subcarrier divided access schemes. Finally, OFDM can be combined with a code division multiple access scheme [13]. A TDMA scheme is proposed for the European WLAN standard and an FDMA scheme combined with a time-slot structure and a frequencyhopping scheme was proposed for the UMTS radio interface [1]. In Section IV, we evaluate our synchronization scheme for such a hybrid TDMA/FDMA multiple access scheme.

Fig. 2 illustrates this access scheme. The available spectrum is subdivided in bands of adjacent subcarriers (FDMA). Within each of these bands, a TDMA scheme is applied. Users are thus separated both in frequency (each user is allocated to a particular subband) and in time (each user is to allocated a particular time slot). In our target system, the minimum access entity is 22 adjacent subcarriers during three consecutive OFDM symbols (see Fig. 2), as will be explained in Section IV.

\section{B. Synchronization Requirements}

Accurate demodulation and detection of an OFDM signal requires subcarrier orthogonality. Variations of the carrier oscillator, the sample clock, or the symbol clock affect the orthogonality of the system (see [8], [14], and [15]). Whereas sample clock variations below $50 \mathrm{ppm}$ have little effect on 


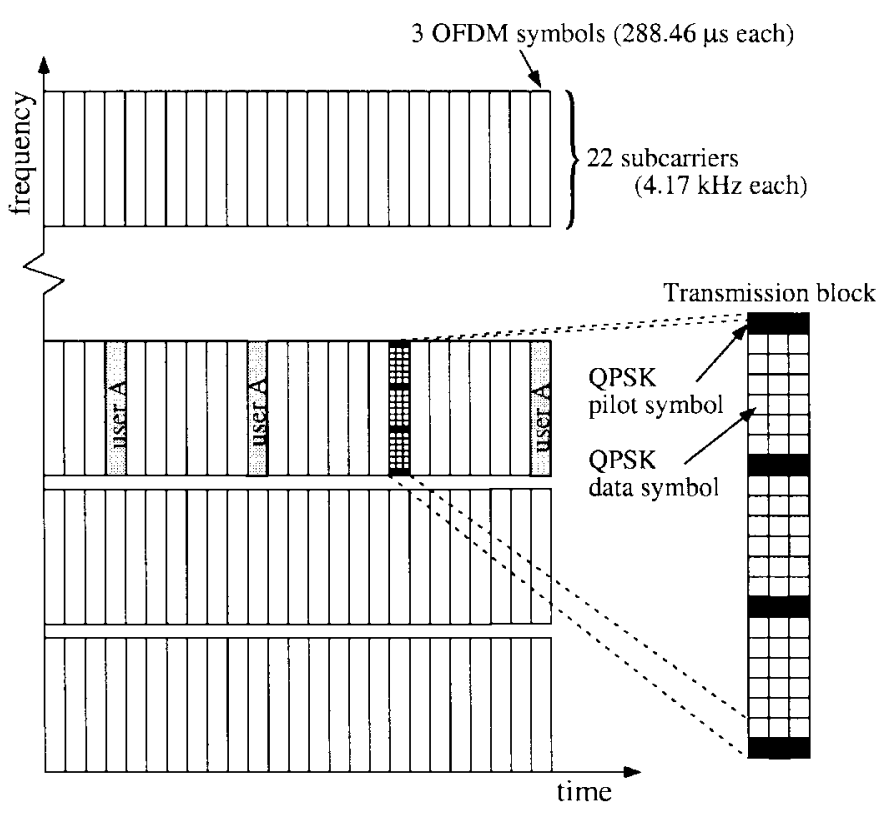

Fig. 2. Time-frequency grid for the UMTS scenario. The users are assigned transmission blocks consisting of 22 adjacent subcarriers and three adjacent OFDM symbols. Each transmission block contains pilot symbols supporting the channel estimation.

the system performance [14], symbol time and frequency offsets may cause intersymbol interference (ISI) and intercarrier interference (ICI) [8], [15] and must usually be counteracted. Therefore, we assume that the sample clocks of the users and the base station are identical (no offset effects), and we focus on a frequency offset and a symbol time offset. We separately consider their effects on the system performance.

The effect of a symbol time offset is the following (assume first perfect carrier frequency synchronization). The demodulator FFT at the base station processes blocks of $N$ samples in the FFT. If different users' transmitted signals are not time-aligned, ISI and ICI (or in a multiuser system: interuser interference) appear at the FFT outputs. Fig. 3 illustrates this interference caused by one user being misaligned with the others. Note first that since we assume perfect sample clocks, such an offset is modeled by an integer-valued number of samples. Note also that the receiver at the base station may identify (and as we will see estimate) each user's time offset, but has no simple means to counteract this offset without becoming misaligned with other users. This is the particular synchronization problem distinguishing broadcast and multiuser synchronization. We focus on the unknown integer-valued time offset $\theta$ of a user's symbol clock: that is, how much this user is misaligned with the block of $N$ samples the receiver processes in the FFT.

Consider a system in which the cyclic prefix is longer than the length of the channel impulse response. Such extra overhead provides robustness against symbol time offsets: as long as a symbol time offset is shorter than the difference between the length of the cyclic prefix and the length of the channel impulse response, the cyclic appearance of the OFDM symbol is preserved, and the offset appears as a linear phase across the subcarrier FFT outputs [16]. In a coherent system, this effect is identified by the channel estimator,

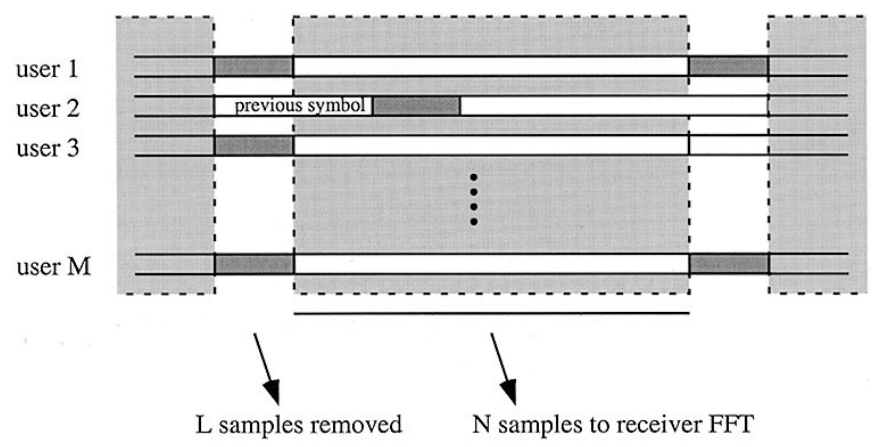

Fig. 3. How one user being misaligned in time with the other users affects the receiver demodulation.

which does not distinguish between phase shifts introduced by the radio channel and those introduced by symbol timing delays [15]. Therefore, the channel equalizer provides the fine synchronization, see also [11]. The requirements on the coarse synchronizer in a coherent system are thus determined by the number of samples the cyclic prefix exceeds the length of the channel impulse response. This provides a system designer with a trade-off tool: by sacrificing data rate, a longer cyclic prefix relaxes the requirements of the symbol synchronizer.

A carrier frequency offset (assuming perfect symbol synchronization) causes a loss of orthogonality between the subcarriers resulting in ICI [8], [17]. We assume that within a user's group of subcarriers, the frequency offset is constant across all tones. This also assumes that in the acquisition mode, some coarse frequency adjustment has been made and we are left with fine-frequency adjustment, i.e. the frequency offset is a fraction of the subcarrier spacing. In [17], it is shown that the effect of a frequency offset is threefold. First, the amplitudes of the FFT outputs are reduced. Secondly, as with symbol time offsets, one user's frequency misalignment with the base station causes the subcarriers to loose their orthogonality resulting in ICI. These first two effects cause a loss of the effective SNR and are hard to counteract.

A third effect of a frequency offset is a common rotation of the subcarriers [12]. This effect will be recognized by a channel estimator, which does not distinguish between phase offsets caused by the channel and those caused by a frequency offset. Thus, a channel equalizer appears also to have fine frequency synchronization capabilities (see also [12]). The analysis of multiuser OFDM systems in [8] shows that a frequency accuracy of $1 \%-2 \%$ of the intercarrier spacing is necessary to obtain a signal-to-interference ratio (SIR) of $30 \mathrm{~dB}$ or higher. A frequency offset may be estimated at the receiver. However, as for symbol time offsets, an adjustment of the receiver base station oscillator would cause the misalignment in frequency with other users.

\section{A MultiUSER SYNCHRONIZATION SCHEME}

Synchronization in broadcast OFDM systems has been investigated in [4] and [6] for instance, and it is in most cases identified with the actual estimation of the offsets. Although synchronization in the downlink yields some difficulties, the uplink in a multiuser system is a more challenging task. In this 


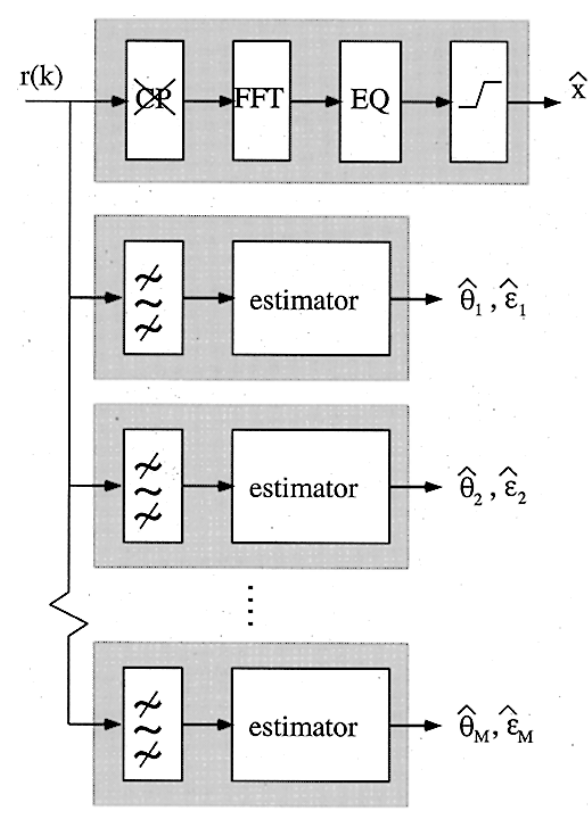

Fig. 4. The structure of the receiver at the base station. One path serves the detection of the data, while other paths estimate the time and frequency offsets for each user.

section, we propose a tracking scheme based on a multiuser time and frequency offset estimator and a downlink control channel on which estimates are fed back to the mobiles.

\section{A. Base Station Receiver Structure and Control Channel}

In Fig. 4, the receiver structure of the base station is shown. It consists of two parts. In one part, the cyclic prefix is removed, and the data are demodulated by means of an FFT. In our target system, the demodulated symbols are equalized by a one-tap channel equalizer and fed into the detector. The channel equalizer also compensates for small time offsets and some of the effects of a frequency offset as discussed earlier. In systems employing convolutional coding, the channel estimates may also be used by the decoder for metric calculation. The second part of the base station receiver serves to track the users' time and frequency offsets. We need to separate one user from another to estimate a given user's time and frequency offset. Grouping the subcarriers together makes separation by filters possible and ensures that within a user's group of subcarriers, there will be little ICI. Subcarriers within the user group may experience ICI from another user group's subcarriers whose time and frequency offset is different. This ICI will be strongest at the outermost tones and will be mitigated by the use of guard intervals. The received sampled baseband signal is fed into a bank of filters, each selecting the frequencies of one band of adjacent subcarriers. This filter bank may be efficiently implemented by means of polyphase filters [18]. Depending on the filter characteristics, a guard carrier may be used between adjacent frequency bands. Each filter output roughly represents one user's signal from which time and frequency offsets can be estimated.

The important difference between the broadcast and multiuser synchronization is how symbol and frequency synchronization is accomplished. In a broadcast or downlink scenario, offsets are estimated by the mobile receiver. These offset estimates then control the adjustments of the local symbol clock and demodulation oscillator. Synchronization thus takes place at the receiver. In the uplink, on the other hand, time and frequency offset estimation is performed in the base station but the clock and oscillator adjustments are made in the user's transmitter. Since all users must arrive at the base station aligned in time and frequency in order to maintain the orthogonality between the subcarriers, all users adapt to the base station's receiver clock and oscillator by adjusting their oscillators and scheduling their transmission according to the base station information.

Therefore, in our scheme, for every connected user a control channel is embedded in the downlink on which control information based on the offset estimates is transmitted back to the user. With the aid of these control parameters, the user aligns its transmitted signal to the receiver reference symbol clock and to the receiver oscillator. The control channel is embedded in the downlink frequency band, which may have a similar multiuser structure as the uplink band and is set up during the initial phase of the connection. Apart from offset estimates, other control parameters for one user include, for instance, which time slots and subbands must be used for the uplink transmission and which transmission power must be applied. Successful tracking synchronization of the user thus relies on the control channel.

\section{B. Time and Frequency Offset Estimation}

1) ML Time and Frequency Estimator for Multiuser OFDM: The estimation of time and frequency offsets is addressed in a number of contributions, e.g., [4], [6], [7], and [17]. As a key part of our synchronization scheme, we propose an estimator based on the concept in [4] which works without the aid of pilot symbols and is independent of the modulation of the carriers. In this concept, statistical redundancy in the received signal, introduced by the cyclic prefix, provides the information about the offsets. Our estimator modifies this concept to suit the multiuser and fading channel environment.

Consider one OFDM symbol received by the base station. Assume that the $N$ subcarriers constituting this symbol are subdivided in $M$ bands of subcarriers, the indexes of which we collect in the set $\mathcal{M}_{m}$. One transmitted OFDM symbol in the $m$ th band of subcarriers is

$$
s_{m}(t)=\sum_{n \in \mathcal{M}_{m}} x_{n} e^{j 2 \pi n t / N T}, \quad-T_{g}<t<N T
$$

where $N T$ is the duration of the OFDM symbol without the cyclic prefix, and $T_{g}$ is the length of the cyclic prefix. We associate with the $m$ th transmitted signal a time offset $\theta_{m}$ relative to the receiver symbol clock and a frequency offset $\varepsilon_{m}$ relative to the receiver demodulation frequency. We consider at the base station the sampled received OFDM signal and model the received signal as

$$
\begin{aligned}
r(k) & =\sum_{m=0}^{M-1} r_{m}(k) \\
& =\sum_{m=0}^{M-1} s_{m}\left(k-\theta_{m}\right) e^{j 2 \pi \varepsilon_{m} k / N}+n_{m}(k)
\end{aligned}
$$




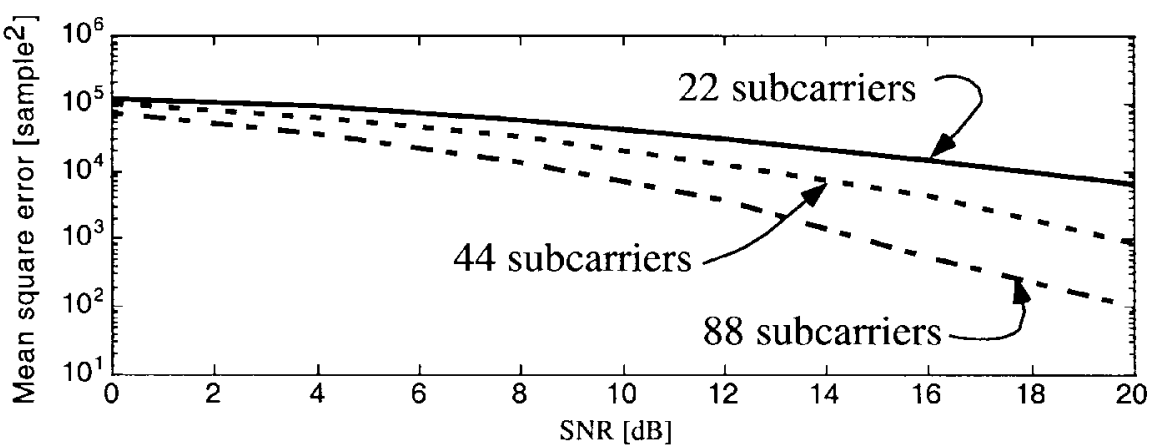

(a)

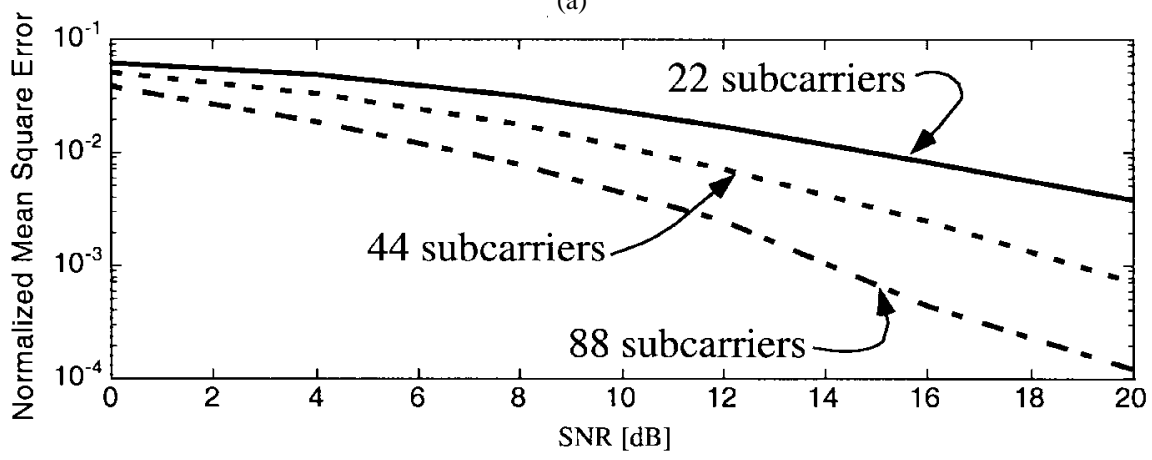

(b)

Fig. 5. The effect of the number of subcarriers in one subband on the mean-squared error of (a) the time offset estimator and (b) the frequency offset estimator. The target system consists of 1024 subcarriers, and the subbands consist of 22 subcarriers (solid), 44 subcarriers (dashed), and 88 subcarriers (dash-dotted).

where the $s_{m}(k)$ is the transmitted signal. The previous model focuses on the frequency or subband division property of the multiple access scheme as this property significantly affects the offset estimation. The receiver offset estimator addresses the time-division property of our target system by applying one estimator to every time slot.

The bank of bandpass filters in the receiver, discussed in Section III-A, separates the users' signals. Note that the signals $r_{m}(k)$ spectrally overlap and that even the use of ideal brickwall filters in the filterbank does not perfectly separate these bands. Perfect separation of the users typically is accomplished by the removal of the cyclic prefix and the demodulation by the FFT. Such separation, however, removes the redundancy that is needed by the offset estimation in our synchronization scheme. Therefore, we use bandpass filters to separate the subcarriers groupings. We will see that the filters separate different users' signals sufficiently for our estimation purpose.

We apply the following estimator (analyzed below) to the outputs of the $m$ th filter [4]

$$
\begin{aligned}
& \hat{\theta}_{m}=\arg \max _{\theta}\left\{\left|\gamma_{m}(\theta)\right|-\rho_{m} \Phi_{m}(\theta)\right\} \\
& \hat{\varepsilon}_{m}=\frac{-1}{2 \pi} \angle \gamma_{m}\left(\hat{\theta}_{m}\right)
\end{aligned}
$$

where

$$
\begin{aligned}
\gamma_{m}(\theta) & =\sum_{k=\theta}^{\theta+L-1} r_{m}^{*}(k) r_{m}(k+N) \\
\Phi_{m}(\theta) & =\frac{1}{2} \sum_{k=\theta}^{\theta+L-1}\left|r_{m}(k)\right|^{2}+\left|r_{m}(k+N)\right|^{2}
\end{aligned}
$$

where $\rho_{m}=\left(\mathrm{SNR}_{m} / \mathrm{SNR}_{m}+1\right)$, and $\mathrm{SNR}_{m}=\left(\sigma_{s_{m}}^{2} / \sigma_{n_{m}}^{2}\right)$. Estimator (4) exploits the correlation introduced by the cyclic prefix to estimate the offsets (see [4]). Its strength is that it is independent of the modulation and that it does not need pilot symbols. It is a one-shot estimator in the sense that the estimates are based on the observation of a single OFDM symbol.

2) ML Estimator Analysis: This estimator is shown to be the joint maximum likelihood (ML) estimate of $\theta$ and $\varepsilon$ [4] if the output of each filter can be written as

$$
r(k)=\tilde{s}(k-\theta) e^{j 2 \pi \varepsilon k / N}+n(k)
$$

where the samples $\tilde{s}(k)$ are Gaussian distributed and uncorrelated except for the pairs of identical samples contained in the cyclic prefix. In our multiuser scenario, the $m$ th transmitted signal is a narrowband signal and has correlation in time in addition to the correlation between pairs of points in the cyclic prefix. This correlation in time was not modeled in the derivation of the time and frequency offset algorithm [4] and is the main source of degradation in our estimator.

The performance of estimator (4) is shown in Fig. 5. There is only one user present in the system in this simulation so the degradation shown is due solely to the increased correlation in time due to the narrowband signal.

Note that the estimator's performance is very sensitive to the number of subcarriers in one subband. This is because the time and frequency offset estimator was designed to operate in an AWGN channel on signals that are uncorrelated outside the cyclic prefix. Using only a small subset of tones violates the assumption of uncorrelated signals. How correlated the data is depends on the number of tones. To see how correlated the 
time-sampled signal is, consider

$$
s_{k}=\sum_{i=M_{0}}^{M+M_{0}-1} x_{i} e^{j 2 \pi k i / N}
$$

where $M$ is the number of adjacent subtones, and $M_{0}$ specifies which group of adjacent subcarriers is used. We find the correlation function for $s_{k}$ to be

$$
\begin{aligned}
E\left[s_{k} s_{k+l}^{*}\right]= & E\left[\sum_{i=M_{0}}^{M+M_{0}-1} x_{i} e^{j 2 \pi k i / N}\right. \\
& \left.\cdot \sum_{n=M_{0}}^{M+M_{0}-1} x_{n}^{*} e^{-j 2 \pi(k+l) n / N}\right] \\
= & \mathcal{E}_{x} \sum_{i=M_{0}}^{M+M_{0}-1} e^{-(j 2 \pi l i / N)} \\
= & \mathcal{E}_{x} e^{-\left(j \pi\left(2 M_{0}+M-1\right) l / N\right)} \frac{\sin \left(\frac{\pi M l}{N}\right)}{\sin \left(\frac{\pi l}{N}\right)}
\end{aligned}
$$

where $\mathcal{E}_{x}=E\left\{\left|x_{i}\right|^{2}\right\}$. From (9), we can see that the larger $M$ is, the smaller the correlation between time-samples.

To see how this correlation affects the time-offset estimator is difficult. However, we can simplify the analysis by instead considering the time-offset estimator $\hat{\theta}_{m}=\arg \max _{\theta}\{\Lambda(\theta)\}$, where $\Lambda(\theta)=\operatorname{Real}(\gamma(\theta))-\rho \Phi(\theta)$. This is the ML estimator when the frequency offset $\epsilon$ is zero in an AWGN channel [4]. This estimator typically will have better performance than the ML estimator in [4], but has similar behavior to the time-offset estimator under consideration.

We will make a mistake when $\Lambda\left(\theta+K_{0}\right)>\Lambda(\theta)$. We will first find the probability that $\Lambda\left(\theta+K_{0}\right)>\Lambda(\theta)$ when there is no correlation between time samples (outside the cyclic prefix). Then we will examine the case for arbitrary correlation between time samples. We will find that the probability that $\Lambda\left(\theta+K_{0}\right)>\Lambda(\theta)$ will be smaller for the case of no correlation between time samples (outside the cyclic prefix). This indicates that the timing metric is more likely to make a mistake when there is correlation between time samples.

From Fig. 5, we note that even for large SNR, reducing the number of tones increases the time-offset estimator meansquared error. So we will further simplify our analysis by assuming that the SNR is large, and hence $\rho \approx 1$. Then a good approximation for this estimator is

$$
\Lambda(\theta) \approx-\sum_{k=\theta}^{\theta+L-1} \frac{1}{2}\left|r_{k}-r_{k+N}\right|^{2} .
$$

This is a form of the time-offset estimator that was proposed in [16]. Given that $\theta$ is the "true" starting point of the OFDM symbol, a synchronization error is made if $\Lambda\left(\theta+K_{0}\right) \geq \Lambda(\theta)$ for some nonzero integer $K_{0}$. This will occur when

$$
\sum_{k=\theta+K_{0}}^{\theta+L-1+K_{0}}\left|r_{k}-r_{k+N}\right|^{2}<\sum_{k=\theta}^{\theta+L-1}\left|r_{k}-r_{k+N}\right|^{2} .
$$

If we subtract the common parts, we can rewrite this as

$$
\sum_{k=\theta+L}^{\theta+L-1+K_{0}}\left|r_{k}-r_{k+N}\right|^{2}<\sum_{k=\theta}^{\theta+K_{0}-1}\left|r_{k}-r_{k+N}\right|^{2}
$$

which is equivalent to

$$
\sum_{k=\theta+L}^{\theta+L-1+K_{0}}\left|r_{k}-r_{k+N}\right|^{2}<\sum_{k=\theta}^{\theta+K_{0}-1}\left|n_{k}-n_{k+N}\right|^{2} .
$$

For the case where the time-samples are uncorrelated (with an AWGN channel and using all the tones), both the left-hand and the right-hand side of (11) are $\chi_{2 K_{0}}^{2}$ random variables [19]. This probability is calculated in Appendix A and is

$$
\begin{aligned}
& P\left(\Lambda\left(\theta+K_{0}\right)>\Lambda(\theta)\right) \\
&=\sum_{m=0}^{K_{0}-1}\left(\begin{array}{c}
K_{0}-1+m \\
m
\end{array}\right)\left(\frac{\mathcal{E}_{x}+\mathcal{N}_{0}}{\mathcal{E}_{x}+2 \mathcal{N}_{0}}\right)^{m} \\
& \cdot\left(\frac{\mathcal{N}_{0}}{\mathcal{E}_{x}+2 \mathcal{N}_{0}}\right)^{K_{0}}
\end{aligned}
$$

which has the same form as the probability of error for a Rayleigh-fading channel with $K_{0}$-path diversity [20]. So given an OFDM symbol using a full set of tones in an AWGN channel, it is less probable that $\Lambda\left(\theta+K_{0}\right)>\Lambda(\theta)$ as $\left|K_{0}\right|$ increases.

However, when the data is correlated, there will be less "diversity" in the data, and it will be more probable that $\Lambda\left(\theta+K_{0}\right)>\Lambda(\theta)$ for larger $\left|K_{0}\right|$. In this case, the probability is no longer $\chi_{2 K_{0}}^{2}$. However, we can still find the probability that $\Lambda\left(\theta+K_{0}\right)>\Lambda(\theta)$ using an analysis similar to that found in analyses of diversity for correlated fading [21]. As in [21], the amount of diversity depends on the rank and relative size of the eigenvalues of the correlation matrices. That is, given a set of correlated zero-mean complex Gaussian random variables, $\mathbf{z}=\left(z_{1}, \ldots, z_{n}\right)^{T}$ with correlation matrix, $R_{z z}$, the pdf of the random variable

$$
w=\sum_{i=1}^{N}\left|z_{i}\right|^{2}
$$

has the same pdf as the random variable

$$
w^{\prime}=\sum_{i=1}^{n} \lambda_{i}\left|u_{i}\right|^{2}
$$

where $\lambda_{i}$ are the eigenvalues of $R_{z z}$ and $u_{i}$ are unit-norm zero-mean complex Gaussian random variables [21].

Now, in our analysis we need to find the pdf of $\Sigma_{\theta+L}^{\theta+L+K_{0}-1}\left|r_{k}-r_{k+N}\right|^{2}$ and $\Sigma_{\theta}^{\theta+K_{0}-1}\left|n_{k}-n_{k+N}\right|^{2}$. If we ignore any correlation between $r_{k}$ and $r_{k+N}$, then $r_{k}-r_{k+N}$ is a zero-mean complex Gaussian random variable with variance $2\left(\mathcal{E}_{x}+\mathcal{N}_{0}\right)$. (When $k$ is outside the cyclic prefix, $r_{k}$ and $r_{k+N}$ belong to different OFDM symbols. We assume that any correlation between $r_{k}$ and $r_{k+N}$ due to filtering is too small to be considered.) Similarly, we can ignore any correlation between $n_{k}$ and $n_{k+N}$ and treat $n_{k}-n_{k+N}$ as a zero-mean complex Gaussian random variable with variance $2 \mathcal{N}_{0}$. Based on this, we can find the probability density function (pdf) of 


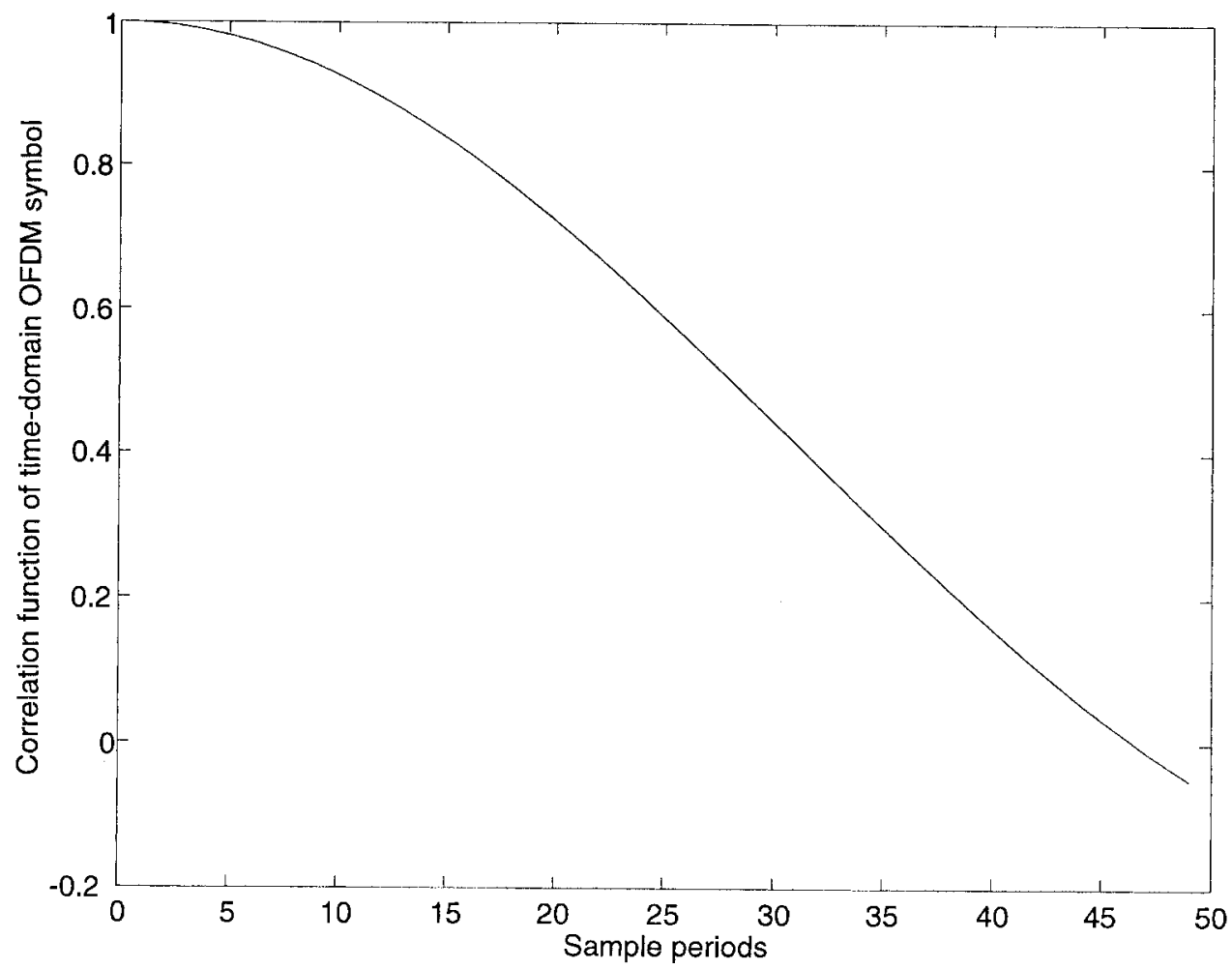

Fig. 6. Correlation between time-samples of an OFDM symbol when using 22 out of 1024 tones.

the two sums if we know the eigenvalues of the correlation matrices

$$
\begin{aligned}
& R_{r r, K_{0}}=E\left[\left(\Delta r_{\theta+L}, \ldots, \Delta r_{\theta+L+K_{0}-1}\right)^{H}\right. \\
& R_{n n, K_{0}}=E\left[\left(\Delta r_{\theta+L}, \ldots, \Delta r_{\theta+L+K_{0}-1}\right)\right] \\
& {\left[\left(\Delta n_{\theta}, \ldots, \Delta n_{\theta+K_{0}-1}\right)^{H}\right.} \\
&\left.\cdot\left(\Delta n_{\theta}, \ldots, \Delta n_{\theta+K_{0}-1}\right)\right]
\end{aligned}
$$

where $\Delta r_{i}=r_{i}-r_{i+N}$ and $\Delta n_{i}=n_{i}-n_{i+N}$. Given the previous argument, we find that

$$
\begin{aligned}
P\left(\Lambda\left(\theta+K_{0}\right)\right. & >\Lambda(\theta)) \\
& =P\left(\sum_{i=0}^{K_{0}-1} \lambda_{r, i}\left|u_{i}\right|^{2} \geq \sum_{i=0}^{K_{0}-1} \lambda_{n, i}\left|v_{i}\right|^{2}\right)
\end{aligned}
$$

where $\lambda_{r, i}$ and $\lambda_{n, i}$ are the eigenvalues of $R_{r r, K_{0}}$ and $R_{n n, K_{0}}$, respectively, and $u_{i}$ and $v_{i}$ are zero-mean independent identically distributed (i.i.d.) complex Gaussian random variables. $\sum_{i=0}^{K_{0}-1} \lambda_{r, i}\left|u_{i}\right|^{2}$ is the weighted sum of independent exponentially distributed random variables. The pdf of this random variable can be found using, for example, characteristic functions. As in diversity arguments, how small (13) is depends on the size and number of eigenvalues of $R_{r r}, K_{0}$ and $R_{n n, K_{0}}$. This probability is calculated in Appendix A for $K_{0}=2$ for the case where the data and the noise have the same correlation function.

To see how much the correlation can affect the timing metric, consider the following example. In our proposed system, we have subdivided 1024 available tones into subgroups of 22 tones each. In this case, the correlation between data symbols will be as shown in Fig. 6 .
Assuming that the noise has the same correlation function as the data signal, then

$$
\begin{aligned}
& R_{r r, K_{0}} \\
& =2\left(\mathcal{E}_{x}+\mathcal{N}_{0}\right) \\
& \left.\begin{array}{cc}
1 & e^{j \phi} \frac{\sin \left(\frac{\pi 22}{1024}\right)}{22 \sin \left(\frac{\pi}{1024}\right)} \\
e^{-j \phi} \frac{\sin \left(\frac{\pi 22}{1024}\right)}{22 \sin \left(\frac{\pi}{1024}\right)} & 1 \\
1 & e^{j \phi} 0.999 \\
e^{-j \phi} 0.999 & 1
\end{array}\right)
\end{aligned}
$$

The eigenvalues of $R_{r r, K_{0}}$ are $\lambda_{r, 0}=3.999\left(\mathcal{E}_{x}+\mathcal{N}_{0}\right)$ and $\lambda_{r, 1}=0.0015\left(\mathcal{E}_{x}+\mathcal{N}_{0}\right)$, while the eigenvalues of $R_{n n, K_{0}}$ are $\lambda_{n, 0}=3.999 \mathcal{N}_{0}$ and $\lambda_{n, 1}=0.0015 \mathcal{N}_{0}$. Using the formula in Appendix A, we find that the probability that $\Lambda(\theta+2)>\Lambda(\theta)$ is equal to 0.08 given an SNR of $10 \mathrm{~dB}$. For uncorrelated random variables, we find using (12) that the probability $\Lambda(\theta+2)>\Lambda(\theta)$ is equal to 0.02 . This means that for correlated data, it is four times as likely that $\Lambda(\theta+2)>\Lambda(\theta)$ than it would be for uncorrelated data.

The frequency offset estimator depends heavily on the timing offset estimator. That is, if the timing offset estimator in our joint frequency and time-offset estimator is incorrect, this means that frequency offset estimator is not acting on the optimal set of points. So any error in the time-offset estimator will also affect the frequency offset estimator. 


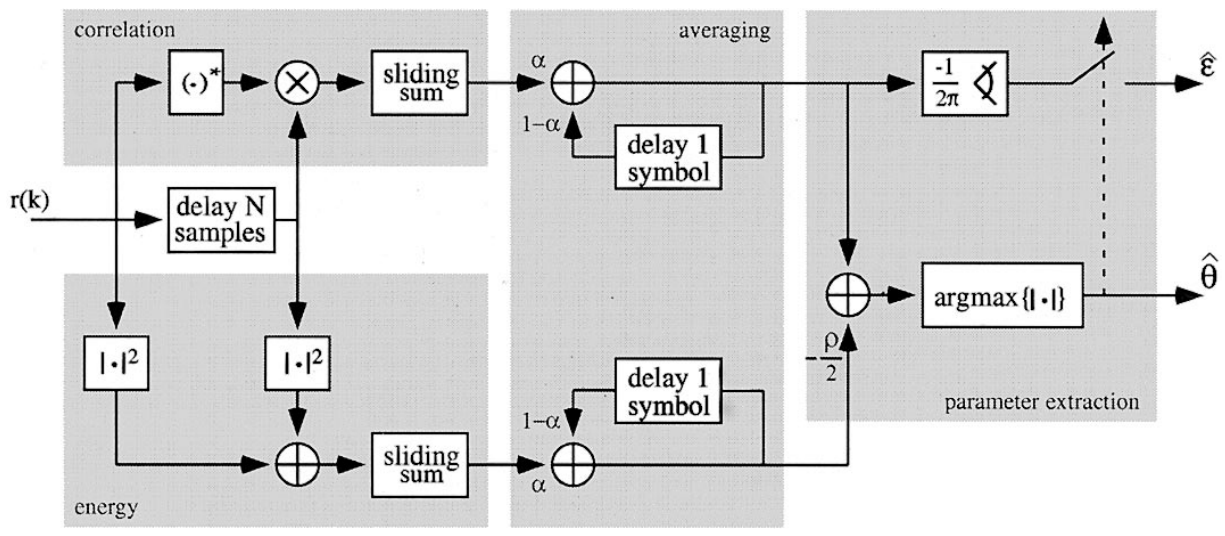

Fig. 7. The structure of the time and frequency offset estimator.

3) Modified Estimator for Multiuser OFDM: Typically in a pure TDMA scenario (only one subband consisting of all subcarriers), the estimator (4) can be applied directly. However, in a system with many narrow subbands the performance of the estimator does not meet the requirements. In addition to the fact that the signal is narrowband, in a multiuser scenario, the filters do not perfectly separate the various users, and a user could also experience multiple access interference. This can also reduce the performance of the estimator. However, in a tracking mode, the amount of ISI and ICI from other users due to time offsets is small as the cyclic prefix of this system is relatively long. In addition, as long as the frequency offset of the users is less than $4 \%$, ICI between the users will also be small. In our simulations, we have found that this is not a problem, though could be a problem in the acquisition mode where time and frequency offsets are expected to be larger.

We now assume that the time and frequency offsets do not change very much between two clock and oscillator updates. The characteristics of the clocks and oscillators may affect the choice of the update frequency in a system. We filter the estimates from (4) using a one-pole infinite impulse response (IIR) filter [22]

$$
\begin{gathered}
\bar{\gamma}_{t}(\theta)=\alpha \bar{\gamma}_{t-1}(\theta)+(1-\alpha) \gamma_{t}(\theta) \\
\bar{\Phi}_{t}(\theta)=\alpha \bar{\Phi}_{t-1}(\theta)+(1-\alpha) \Phi_{t}(\theta)
\end{gathered}
$$

where $0 \leq \alpha \leq 1$ is a forgetting factor and $\gamma_{t}(\theta)$ and $\Phi_{t}(\theta)$ are the one-shot statistics (5). For $\alpha=0$, this estimator is identical to (5), but for other $\alpha$ it exploits information about the offsets carried by past redundancies. In the rest of this paper, we show results for $\alpha=0.75$. Based on this statistic and the estimator structure (4), we use the estimator

$$
\begin{aligned}
& \hat{\theta}_{t}=\arg \max \left\{\left|\bar{\gamma}_{t}(\theta)\right|+\rho \bar{\Phi}_{t}(\theta)\right\} \\
& \hat{\varepsilon}_{t}=\frac{-1}{2 \pi} \angle \bar{\gamma}_{(}\left(\hat{\theta}_{t}\right) .
\end{aligned}
$$

The exponential weighting is suitable in environments where $\theta$ and $\varepsilon$ vary slowly. Fig. 7 shows the structure of the estimator. Estimator (15) weights the one-symbol statistics $\bar{\gamma}_{t}(\theta)$ and $\bar{\Phi}_{t}(\theta)$ exponentially. Such an IIR implementation of the averaging requires the receiver to store only the joint statistic $\bar{\gamma}_{t}(\theta)$ and $\bar{\Phi}_{t}(\theta)$.
TABLE I

Transmission Characteristics OF THE SySTEM

\begin{tabular}{l|l}
\hline Center frequency & $2.2 \mathrm{GHz}$ \\
\hline Uplink / Downlink bandwidth & $4.27 \mathrm{MHz} / 4.27 \mathrm{MHz}$ \\
\hline Intercarrier spacing & $4.17 \mathrm{kHz}$ \\
\hline Symbol length & $240 \mu \mathrm{sec}$ (1024 samples) \\
\hline Cyclic prefix & $48 \mu \mathrm{sec}$ (205 samples) \\
\hline
\end{tabular}

TABLE II

Multiple Access Characteristics of the System

\begin{tabular}{l|l}
\hline Transmission block & 22 subcarriers $\times 3$ symbols \\
\hline Data rate & multiples of $5.7 \mathrm{kbits} / \mathrm{second}$ \\
\hline Minimum data rate (Speech service) & $17.1 \mathrm{kbits} /$ second \\
\hline Maximum data rate & $5 \mathrm{Mbits} /$ second \\
\hline
\end{tabular}

\section{Simulation Results}

\section{A. Multiuser Scenario}

Table I specifies the transmission parameters of our target multiuser system based on [1], which we will use to evaluate our synchronization scheme. The length of the cyclic prefix is sufficiently long to provide immunity against channel dispersion. The length of the cyclic prefix was chosen, in part, to make the OFDM symbol size compatible with existing GSM systems [1], [3], half a GSM time slot.

The prefix accommodates both the delay spread of the channel and offsets of the symbol clock. As long as the accumulated effect of a symbol clock offset and a far echo in the channel impulse response does not exceed $48 \mu \mathrm{s}$, the system performance is equal to a system with a perfect symbol clock. As discussed in Section II-B, the cyclic prefix thus provides robustness to small symbol time offsets. For example, if the channel impulse response contains echoes up to 34 $\mu \mathrm{s}$, the symbol synchronizer must provide synchronization to within the remaining $14 \mu \mathrm{sec}$ (60 samples). In Section IV, we will see that our synchronization scheme very rarely exceeds these $14 \mu$ s (60 samples).

Table II specifies and Fig. 2 illustrates the multiple access characteristics of our target system [1], already briefly dis- 
cussed in Section II. As a minimal access entity, every user is assigned 22 adjacent subcarriers during three consecutive OFDM symbols. We will refer to such an entity as a transmission block. In between two subbands are two guard carriers which are not modulated.

For a typical low-rate circuit service, such as a speech service, three such transmission blocks are transmitted every $18.47 \mathrm{~ms}$. For higher data rates, users request more transmission blocks. The proposal [1] provides more frequency diversity via a slow frequency-hopping system, which we do not regard in our simulations. The proposed synchronization scheme would benefit from this hopping scheme.

We evaluate the synchronization scheme for two types of modulation. First, a differential quatenary phase shift keying (QPSK) system modulates the data symbols differentially across the subcarriers. Therefore, 21 information symbols are transmitted in each OFDM symbol in a transmission block. Secondly, a coherent QPSK system is simulated, in which the base station uses a channel estimator [23], [24] and a channel equalizer to correct for the multiplicative channel distortion of the data symbols. The pilot positions for channel estimation are shown in Fig. 2. Each OFDM symbol in a transmission block contains 22 QPSK symbols, four of which are known pilot symbols. This modulation system differs from the proposal [1] which employs differential QPSK (DQPSK).

For the coherent system simulations, the base station channel estimator $\hat{h}_{k}$ is the following. We base our estimator on the four received data symbols at the pilot positions, which we collect in the $4 \times 1$ vector $y_{P}$. The base station then estimates the channel attenuations with the linear minimum mean-square error estimator

$$
\hat{\mathbf{h}}=\mathbf{R}_{h y_{P}} \mathbf{R}_{y_{P} y_{P}}^{-1} \mathbf{y}_{P}
$$

where $\mathbf{R}_{h y_{P}}$ is the cross correlation matrix between the channel $\mathbf{h}$ and the received data $\mathbf{y}_{P}$, and $\mathbf{R}_{y_{P} y_{P}}$ is the autocorrelation matrix of the received pilot data. Since we do not know these channel correlations, we design generic matrices based on the following correlation properties: the assumed channel delay spread is exponentially decaying with an RMS value of ten samples. The channel correlation matrices for this channel are given in [23]. The correlation matrix is designed for an assumed SNR of $20 \mathrm{~dB}$ (see [23]).

\section{B. Channel Model}

We have evaluated the synchronization scheme in one of the channel environments defined by ETSI for the evaluation of UMTS radio interface proposals. The time-varying channel impulse response for these models can be described by

$$
h(\tau, t)=\sum_{i} \alpha_{i}(t) \delta\left(\tau-\tau_{i}\right)
$$

This equation defines the channel impulse response at time $t$ as a function of the lag $\tau$. In this paper, we will evaluate our synchronization algorithm for the choices of $\alpha_{i}$ and $\tau_{i}$ associated with the "Vehicular A" channel environment [25]. The channel taps $\alpha_{i}(t)$ are complex independent stochastic variables, fading with Jakes' Doppler spectrum [26], with a
TABLE III

Characteristics of the ETSi "Vehicular A" Channel Environment

\begin{tabular}{r|r|r}
\hline tap & $\begin{array}{r}\text { relative delay } \\
\text { (nsec) }\end{array}$ & $\begin{array}{r}\text { average power } \\
(\mathrm{dB})\end{array}$ \\
\hline 1 & 0 & 0 \\
\hline 2 & 310 & -1.0 \\
\hline 3 & 710 & -9.0 \\
\hline 4 & 1090 & -10.0 \\
\hline 5 & 1730 & -15.0 \\
\hline 6 & 2510 & -20.0 \\
\hline
\end{tabular}

TABLE IV

Performance of the Time and Frequency Offset Estimates

\begin{tabular}{l|l}
\hline SNR $=10 \mathrm{~dB}$ & \\
\hline$\sqrt{E\left\{|\theta-\hat{\theta}|^{2}\right\}}$ & 6.41 \\
\hline$|\theta-\hat{\theta}|>20$ samples & $0.98 \%$ \\
\hline$|\theta-\hat{\theta}|>30$ samples & $0.08 \%$ \\
\hline$|\theta-\hat{\theta}|>40$ samples & $0.01 \%$ \\
\hline$\sqrt{E\left\{|\varepsilon-\widehat{\varepsilon}|^{2}\right\}}$ & $1.17 \%$ \\
\hline$|\varepsilon-\hat{\varepsilon}|>1 \%$ & $35.22 \%$ \\
\hline$|\varepsilon-\hat{\varepsilon}|>2 \%$ & $8.32 \%$ \\
\hline$|\varepsilon-\hat{\varepsilon}|>3 \%$ & $1.96 \%$ \\
\hline
\end{tabular}

maximum Doppler frequency of $240 \mathrm{~Hz}$, reflecting a mobile speed of approximately $120 \mathrm{~km} / \mathrm{h}$ (and scatterers uniformly distributed around the mobile). The real-valued $\tau_{i}$ and the variance of the complex-valued $\alpha_{i}$ are given in [25] and repeated in Table III.

\section{Estimator Performance}

In our synchronization scheme, we assume that the users' signals arrive at the base station with equal power; that is, we assume ideal power control. The characteristics of the filters determine the sensitivity of our estimator in systems with large power variations between users. The estimates $\hat{\theta}_{m}$ and $\hat{\varepsilon}_{m}$ are fed back to the transmitter ten times/s. The system performance is determined by both the performance of the estimator and the characteristics of the feedback scheme. We first investigate the performance of the joint time and frequency offset estimator (15) employed in the base station, and then we present simulation results for the system performance in Section IV. We consider the low-rate service with three transmission blocks each $18.47 \mathrm{~ms}$.

We evaluate the tracking performance of the estimators. Figs. 8 and 9 show the estimates versus time. The time axis in the figures shows the index of the transmitted OFDM symbol for one user. The true time offset follows a sinusoid with 30 samples amplitude and a period of 96 OFDM symbols. Such rapid time offset variations exceed practical offset variations 


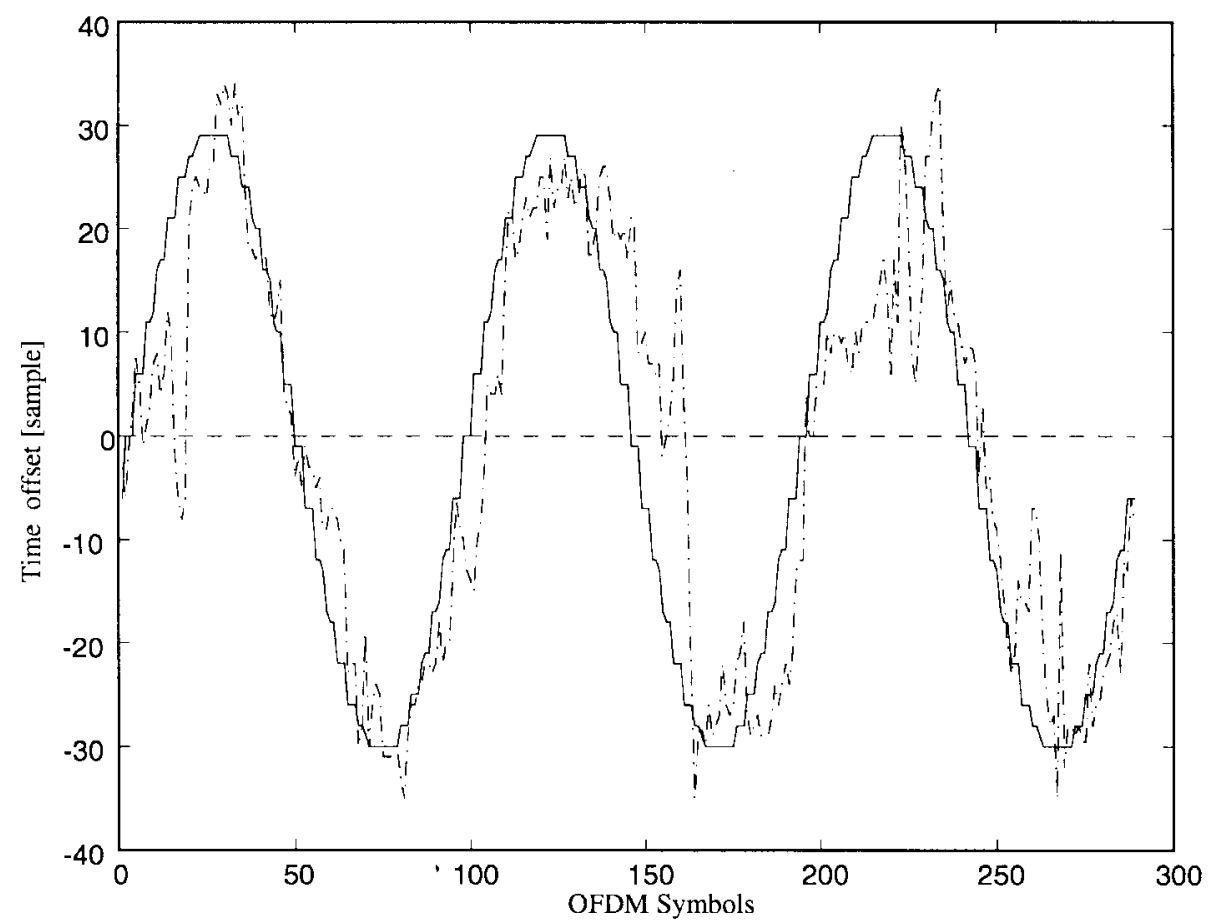

Fig. 8. Timing offsets versus time. The true timing offset (solid) and the estimated timing offset (dashed) at an SNR $=10 \mathrm{~dB}$ and using the ETSI "Vehicular A" channel environment (Table III).

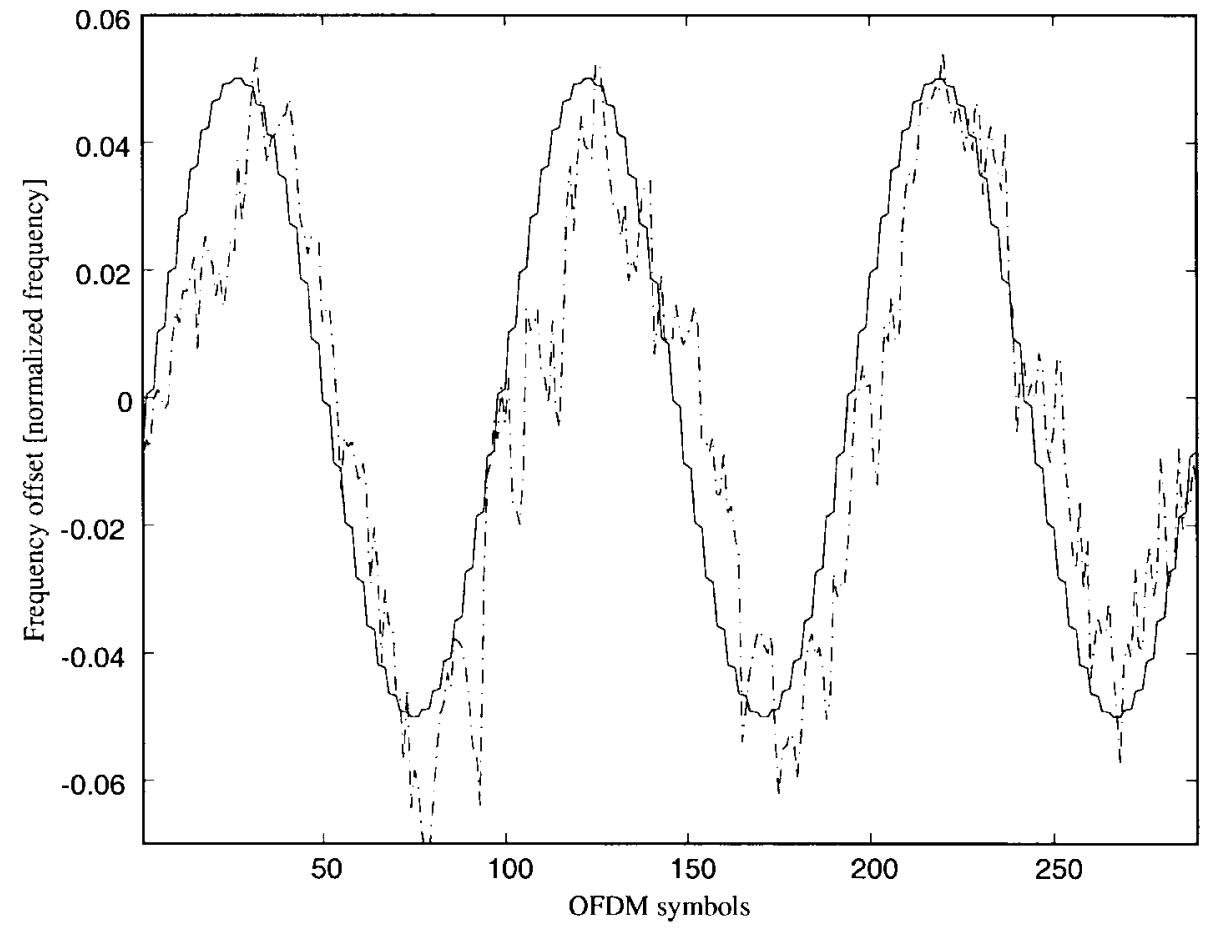

Fig. 9. Frequency offsets versus time. The true frequency offset (solid) and the estimated frequency offset (dashed) at an SNR $=10 \mathrm{~dB}$ and using the ETSI "Vehicular A" channel environment (Table III).

a base station in our UMTS scenario would experience. The purpose of these curves is to show the tracking ability of the estimator. The true frequency offsets also follow a sinusoid with an amplitude of $5 \%$ of the intercarrier spacing. As with the time offsets, such frequency drifts exceed practical drifts and show here the frequency estimator's tracking ability.
The distribution of the estimation error for this run is shown in Fig. 10, while Table IV shows the estimator standard deviations. Of special interest is the percentage of the time offset estimates which fall outside a particular interval. This percentage together with the length of the channel dispersion shows how often ISI will occur. 

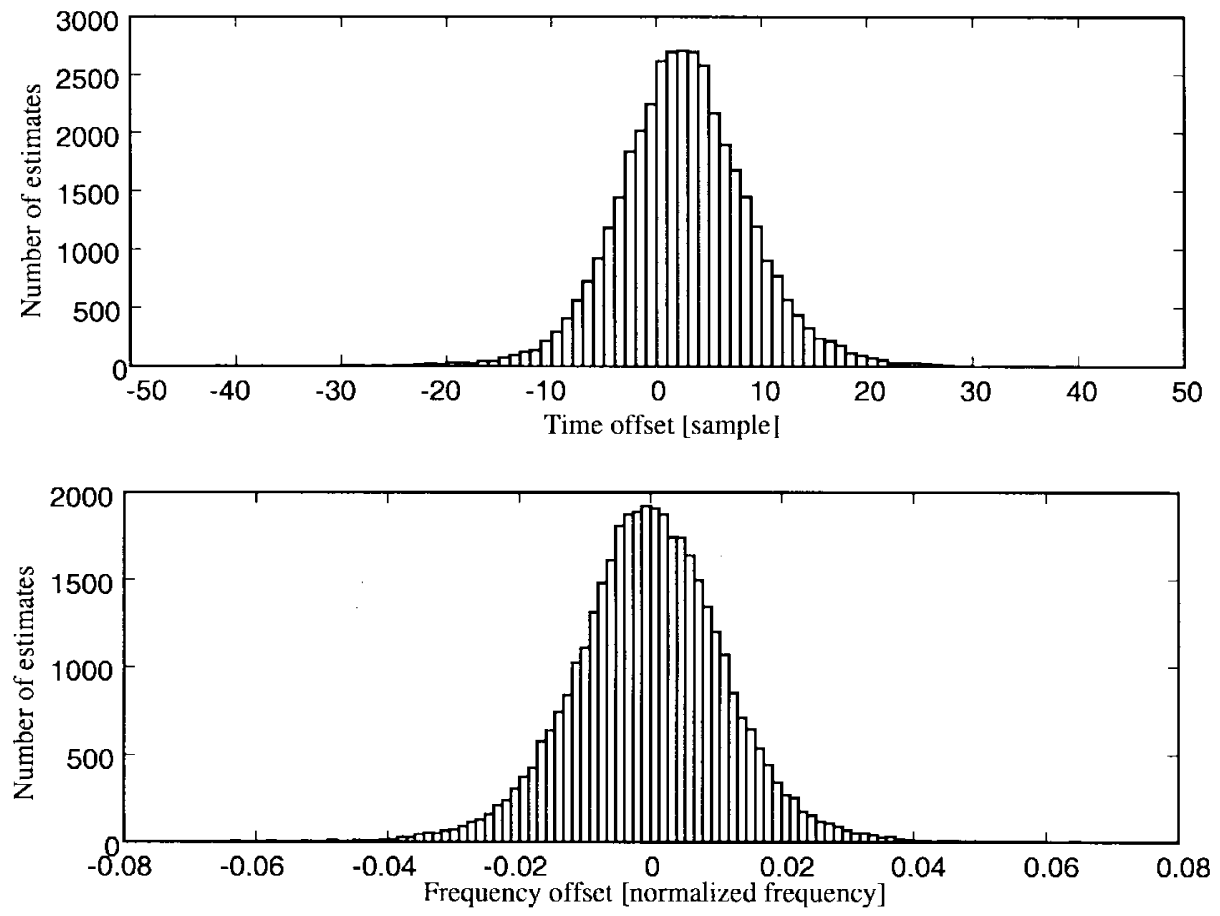

Fig. 10. The distribution of the time and frequency offset estimates.

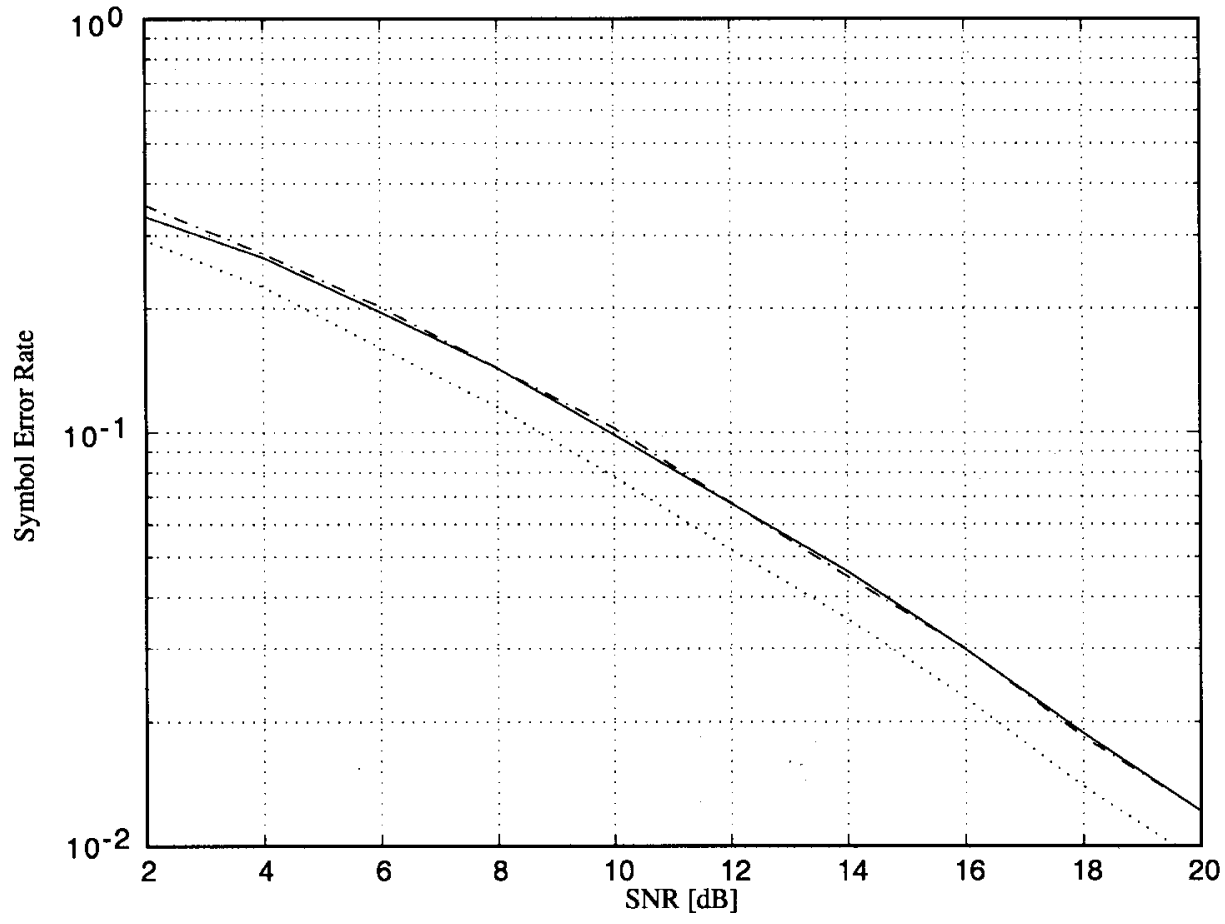

Fig. 11. The uncoded symbol error rate of the UMTS scenario with QPSK modulated subcarriers and channel estimation/equalization. System performance without synchronization errors and known channel (dotted); system performance without synchronization errors but with channel estimation (solid); system performance for the proposed synchronization scheme (dash-dotted). In the simulations, none of the time offset estimates that were fed back to the mobile caused ISI.

\section{System Performance}

In Section III, we have proposed a synchronization scheme and an estimator of the user's time and frequency offset to the base station. Here we evaluate the uplink synchronization performance of the UMTS proposal employing the proposed synchronization scheme.
Fig. 11 shows the uncoded symbol error rate (SER) of the system (employing QPSK on each subcarrier) for a system with perfect synchronization and ideal channel knowledge in the receiver equalizer, as well as the SER for a system employing the channel estimator (16) in the base station receiver and fed back estimates of the time and frequency 


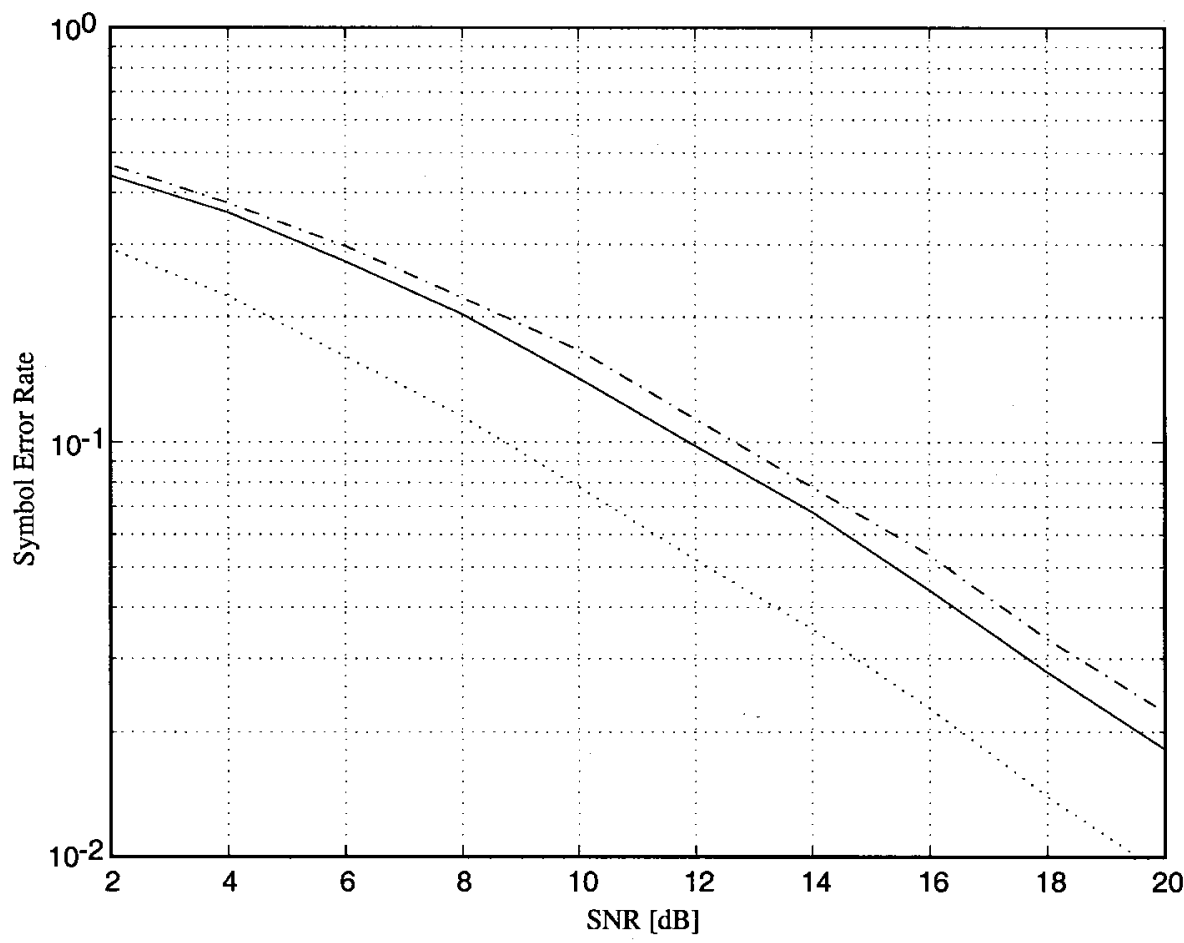

Fig. 12. The uncoded symbol error rate for the UMTS scenario with DPSK modulated subcarriers. System performance without synchronization errors (solid); system performance for the proposed synchronization scheme (dash-dotted); system performance for the system with QPSK modulated subcarriers without synchronization errors and known channel (dotted). In the simulations, none of the time offset estimates that were fed back to the mobile caused ISI.

offsets [using (15)] in the transmitter. In the latter case, we have assumed a clock drift of $10 \mathrm{ppm}$ and that estimates are fed back to the mobile 10 times/s. The loss with respect to the perfectly synchronized system with channel estimation is indistinguishable because the channel estimator compensates for parts of the time and frequency offsets. In the simulations, none of the time offset estimates that were fed back to the mobile caused ISI. For long runs, there may be instances when the time offset and the length of the channel impulse response exceed the cyclic prefix and ISI will occur.

Fig. 12 shows the same curves for a system employing differential PSK across the tones. In this scenario no channel estimation is performed. Besides the $3 \mathrm{~dB}$ loss in SNR compared to the coherent QPSK modulation with perfect channel knowledge, the tracking loop now results in a performance decrease because of time offsets. This is because the phase offset due to misestimation of the timing offset has not been corrected by channel estimation A time-offset misadjustment of say, $\theta_{e}$ samples, results in a phase rotation of $e^{2 \pi \theta_{e} n / N}$ on the $n$th tone. When demodulating the adjacent tone DQPSK symbols, there will be a phase difference of $e^{2 \pi \theta_{e} N}$ between the symbols, resulting in an increase in error. In this case, the effects of a time offset, which appears as a linear phase distortion across the tones, are not compensated for by a channel estimator.

\section{Summary AND CONClusions}

We have shown a way to track different users in the multiuser uplink of an OFDM-based system. Time and frequency offsets are estimated at the base station. Control information, based on these estimates, is returned on a downlink control channel to the user, who adjusts its symbol clock and its local oscillator accordingly. The estimation is done by exploiting statistical redundancy due to the presence of the cyclic prefix.

We have illustrated the synchronization scheme with parameter choices and simulation results for the proposal [1] and show that the synchronization requirements are satisfied. Simulations show that the performance of a coherently modulated system employing our synchronization scheme is not visibly decreased compared to the performance of a system without time and frequency offsets. In a differentially modulated system, the effect of the time errors on the system performance decreases the performance by about $0.7 \mathrm{~dB}$ compared to a system without time and frequency offsets.

\section{APPENDIX A}

\section{A. Derivation of Probability of Error for Uncorrelated Samples}

To find the probability that $\Lambda(\theta)<\Lambda\left(\theta+K_{0}\right)$, we first consider the pdf's of

$$
\sum_{k=0}^{K_{0}-1}\left|r_{k}-r_{k+N}\right|^{2}=z
$$

and

$$
\sum_{k=0}^{K_{0}-1}\left|n_{k}-n_{k+N}\right|^{2}=w
$$


which are $\chi_{2 K_{0}}^{2}$ random variables (when $r_{k}$ and $n_{k}$ are independent complex Gaussian variables) with pdf's [19]

$$
f_{Z}(z)= \begin{cases}\frac{z^{K_{0}-1}}{\left(K_{0}-1\right) !\left(2\left(\mathcal{E}_{x}+\mathcal{N}_{0}\right)\right)^{K_{0}}} & \\ \cdot \exp \left(-\frac{z}{2\left(\mathcal{E}_{x}+\mathcal{N}_{0}\right)}\right), & z \geq 0 \\ 0, & \text { otherwise }\end{cases}
$$

and

$$
f_{W}(w)=\left\{\begin{array}{l}
\frac{w^{K_{0}-1}}{\left(K_{0}-1\right) !\left(2\left(\mathcal{N}_{0}\right)\right)^{K_{0}}} \exp \left(-\frac{w}{2\left(\mathcal{N}_{0}\right)}\right) \\
w \geq 0 \\
0, \quad \text { otherwise }
\end{array}\right.
$$

respectively. Using math tables [27], we can find the equation shown at the bottom of the page.

\section{B. Derivation of Probability of Error for \\ Correlated Samples $\left(K_{0}=2\right)$}

To find the probability that $\Lambda\left(\theta+K_{0}\right) \geq \Lambda(\theta)$ for correlated random variables we use (13) and recognize that the random variable $z=\Sigma_{i=0}^{K_{0}-1} \lambda_{r, i}\left|u_{i}\right|^{2}$ and $w=\Sigma_{i=0}^{K_{0}-1} \lambda_{n, i}\left|v_{i}\right|^{2}$ are both sums of independent $\chi_{2}^{2}$ random variables. For $K_{0}=2$, the characteristic function of $z$ is [21]

$$
\begin{aligned}
\phi_{Z}(f)= & \frac{1}{\left(1+j 2 \pi \lambda_{r, 0}\right)\left(1+j 2 \pi \lambda_{r, 1}\right)} \\
= & \frac{-\lambda_{r, 0}}{\lambda_{r, 1}-\lambda_{r, 0}} \frac{1}{1+j 2 \pi \lambda_{r, 0} f} \\
& +\frac{\lambda_{r, 0}}{\lambda_{r, 1}-\lambda_{r, 0}} \frac{1}{1+j 2 \pi \lambda_{r, 1} f}
\end{aligned}
$$

Assuming the covariance matrix $R_{r r, K_{0}}=2\left(\mathcal{E}_{x}+\right.$ $\left.\mathcal{N}_{0}\right)\left(\begin{array}{ll}1 & \rho \\ \rho^{*} & 1\end{array}\right)$, for some correlation factor $\rho$, we find the pdf of $z$ to be

$$
f_{Z}(z)=\left\{\begin{array}{l}
\frac{-1}{\left(\lambda_{1}-\lambda_{0}\right) 2\left(\mathcal{E}_{x}+\mathcal{N}_{0}\right)} \exp \left(-\frac{z}{\lambda_{0} 2\left(\mathcal{E}_{x}+\mathcal{N}_{0}\right)}\right) \\
+\frac{1}{\left(\lambda_{1}-\lambda_{0}\right) 2\left(\mathcal{E}_{x}+\mathcal{N}_{0}\right)} \exp \left(-\frac{z}{\lambda_{1} 2\left(\mathcal{E}_{x}+\mathcal{N}_{0}\right)}\right) \\
\begin{array}{c}
z \geq 0 \\
0, \quad \text { otherwise }
\end{array}
\end{array}\right.
$$

where $\lambda_{1}$ and $\lambda_{0}$ are the eigenvalues of the correlation matrix. Assuming the noise and the data have the same correlation function and are independent of each other, the pdf of $w$, as defined in as in (19), is

$$
f_{W}(w)=\left\{\begin{array}{l}
\frac{-1}{\left(\lambda_{1}-\lambda_{0}\right)\left(2 \mathcal{N}_{0}\right)} \exp \left(-\frac{w}{\lambda_{0}\left(2 \mathcal{N}_{0}\right)}\right) \\
+\frac{1}{\left(\lambda_{1}-\lambda_{0}\right)\left(2 \mathcal{N}_{0}\right)} \exp \left(-\frac{w}{\lambda_{1}\left(2 \mathcal{N}_{0}\right)}\right) \\
w \geq 0 \\
0, \quad \text { otherwise. }
\end{array}\right.
$$

Then, as in the previous section, we find

$$
\begin{aligned}
P(z \leq & w) \\
= & \int_{0}^{\infty} f_{Z}(z) d z \int_{z}^{\infty} f_{W}(w) d w \\
= & \int_{0}^{\infty} f_{Z}(z) \frac{-\lambda_{0}}{\left(\lambda_{1}-\lambda_{0}\right)} \exp \left(-\frac{z}{\lambda_{0}\left(2 \mathcal{N}_{0}\right)}\right) d z \\
& +\int_{0}^{\infty} f_{Z}(z) \frac{\lambda_{1}}{\left(\lambda_{1}-\lambda_{0}\right)} \exp \left(-\frac{z}{\lambda_{1}\left(2 \mathcal{N}_{0}\right)}\right) d z \\
= & \int_{0}^{\infty} \frac{-1}{\left(\lambda_{1}-\lambda_{0}\right) 2\left(\mathcal{E}_{x}+\mathcal{N}_{0}\right)} \frac{1}{\left(\lambda_{1}-\lambda_{0}\right)} \\
& \cdot \exp \left(-z\left(\frac{1}{\lambda_{0} 2\left(\mathcal{E}_{x}+\mathcal{N}_{0}\right)}+\frac{1}{\lambda_{0}\left(2 \mathcal{N}_{0}\right)}\right)\right) d z \\
& +\int_{0}^{\infty} \frac{-\lambda_{1}}{\left(\lambda_{1}-\lambda_{0}\right) 2\left(\mathcal{E}_{x}+\mathcal{N}_{0}\right)} \frac{1}{\left(\lambda_{1}-\lambda_{0}\right)} \\
& \cdot \exp \left(-z\left(\frac{1}{\lambda_{0} 2\left(\mathcal{E}_{x}+\mathcal{N}_{0}\right)}+\frac{1}{\lambda_{1}\left(2 \mathcal{N}_{0}\right)}\right)\right) d z
\end{aligned}
$$

$$
\begin{aligned}
P(z \leq w)= & \int_{0}^{\infty} f_{Z}(z) d z \int_{z}^{\infty} f_{W}(w) d w \\
= & \int_{0}^{\infty} f_{W}(w) \sum_{r=0}^{K_{0}-1} \frac{w^{K_{0}-1-r}}{\left(K_{0}-1-r\right) !\left(2\left(\mathcal{N}_{0}\right)\right)^{K_{0}-r-1}} \exp \left(-\frac{w}{2\left(\mathcal{N}_{0}\right)}\right) d w \\
= & \sum_{r=0}^{K_{0}-1} \int_{0}^{\infty} \exp \left(-w\left(\frac{1}{2\left(\mathcal{N}_{0}\right)}+\frac{1}{2\left(\mathcal{E}_{x}+\mathcal{N}_{0}\right)}\right)\right) \\
= & \sum_{r=0}^{K_{0}-1} \frac{w^{2 K_{0}-2-r}}{\left(K_{0}-1\right) !\left(K_{0}-1-r\right) !\left(\left(\mathcal{N}_{0}\right)\right)^{K_{0}-r-1}\left(\left(\mathcal{E}_{x}+\mathcal{N}_{0}\right)\right)^{K_{0}}}\left(\frac{\left.\left(2 K_{0}-1\right) !\left(K_{0}-1-r\right) !\left(2\left(\mathcal{N}_{0}\right)\right)^{K_{0}-r-1}+\mathcal{N}_{0}\right)}{\mathcal{E}_{x}+2 \mathcal{N}_{0}}\right)^{2 K_{0}-1-r} \\
= & \sum_{r=0}^{K_{0}-1}\left(\begin{array}{c}
2 K_{0}-r-2 \\
K_{0}-1
\end{array}\right)\left(\frac{\mathcal{N}_{0}}{\mathcal{E}_{x}+2 \mathcal{N}_{0}}\right)^{K_{0}}\left(\frac{\mathcal{N}_{0}+\mathcal{E}_{x}}{\mathcal{E}_{x}+2 \mathcal{N}_{0}}\right)^{K_{0}-r-1} d w \\
= & \sum_{m=0}^{K_{0}-1}\left(\begin{array}{c}
K_{0}-1+m \\
K_{0}-1
\end{array}\right)\left(\frac{\mathcal{N}_{0}}{\mathcal{E}_{x}+2 \mathcal{N}_{0}}\right)^{K_{0}}\left(\frac{\mathcal{N}_{0}+\mathcal{E}_{x}}{\mathcal{E}_{x}+2 \mathcal{N}_{0}}\right)^{m}
\end{aligned}
$$




$$
\begin{aligned}
+ & \int_{0}^{\infty} \frac{-1}{\left(\lambda_{1}-\lambda_{0}\right) 2\left(\mathcal{E}_{x}+\mathcal{N}_{0}\right)} \frac{\lambda_{0}}{\left(\lambda_{1}-\lambda_{0}\right)} \\
\cdot & \exp \left(-z\left(\frac{1}{\lambda_{1} 2\left(\mathcal{E}_{x}+\mathcal{N}_{0}\right)}+\frac{1}{\lambda_{0}\left(2 \mathcal{N}_{0}\right)}\right)\right) d z \\
+ & \int_{0}^{\infty} \frac{1}{\left(\lambda_{1}-\lambda_{0}\right) 2\left(\mathcal{E}_{x}+\mathcal{N}_{0}\right)} \frac{\lambda_{1}}{\left(\lambda_{1}-\lambda_{0}\right)} \\
& \cdot \exp \left(-z\left(\frac{1}{\lambda_{1} 2\left(\mathcal{E}_{x}+\mathcal{N}_{0}\right)}+\frac{1}{\lambda_{1}\left(2 \mathcal{N}_{0}\right)}\right)\right) d z \\
= & \frac{\lambda_{0}^{2}}{\left(\lambda_{1}-\lambda_{0}\right)^{2}} \frac{\mathcal{N}_{0}}{\mathcal{E}_{x}+2 \mathcal{N}_{0}}-\frac{\lambda_{1}^{2}}{\left(\lambda_{1}-\lambda_{0}\right)^{2}} \\
\cdot & \frac{\lambda_{0}^{2}}{\mathcal{E}_{x}+\left(\frac{\lambda_{1}}{\lambda_{0}}+1\right) \mathcal{N}_{0}}-\frac{\lambda_{0}^{2}}{\left(\lambda_{1}-\lambda_{0}\right)^{2}} \\
& \cdot \frac{\mathcal{N}_{0}}{\mathcal{E}_{x}+\left(\frac{\lambda_{0}}{\lambda_{1}}+1\right) \mathcal{N}_{0}}+\frac{\lambda_{1}^{2}}{\left(\lambda_{1}-\lambda_{0}\right)^{2}} \frac{\mathcal{N}_{0}}{\mathcal{E}_{x}+2 \mathcal{N}_{0}} .
\end{aligned}
$$

\section{REFERENCES}

[1] Concept group Beta, "OFDMA Evaluation Report-The multiple access proposal for the UMTS Terrestrial Radio Air Interface (UTRA)," Tdoc/SMG 896/97, ETSI SMG Meeting No. 24, Madrid, Spain, Dec. 1997.

[2] "Broadband Radio Access Networks (BRAN); 'Inventory of broadband radio technologies and techniques'," ETSI Tech. Rep., DTR/BRAN030001, 1998.

[3] M. Mouly and M. B. Pautet, The GSM System For Mobile Communications. France: Palaiseau, 1992.

[4] J. J. van de Beek, M. Sandell, and P. O. Börjesson, "ML estimation of timing and frequency offset in OFDM systems," IEEE Trans. Signal Processing, vol. 45, pp. 1800-1805, July 1997.

[5] T. M. Schmidl, "Synchronization Algorithms for Wireless Data Transmission Using Orthogonal Frequency Division Multiplexing (OFDM)", Ph.D. dissertation, Stanford University, Stanford, CA, June 1997.

[6] W. D. Warner and C. Leung, "OFDM/FM frame synchronization for mobile radio data communication," IEEE Trans. Vehic. Technol., vol. 42, pp. 302-313, Aug. 1993.

[7] P. J. Tourtier, R. Monnier, and P. Lopez, "Multicarrier modem for digital HDTV terrestrial broadcasting," Signal Processing: Image Commun., vol. 5, pp. 379-403, Dec. 1993

[8] L. Wei and C. Schlegel, "Synchronization requirements for multi-user OFDM on satellite mobile and two-path Rayleigh-fading channels," IEEE Trans. Commun., vol. 43, pp. 887-895, Feb./Mar./Apr. 1995.

[9] J. A. C. Bingham, "Multicarrier modulation for data transmission: An idea whose time has come," IEEE Commun. Mag., vol. 28, pp. 5-14, May 1990.

[10] A. Peled and A. Ruiz, "Frequency domain data transmission using reduced computational complexity algorithms," in Proc. IEEE Int. Conf. Acoustics, Speech, Signal Processing, Denver, CO, 1980, pp. 964-967.

[11] L. Hazy and M. El-Tanany, "Synchronization of OFDM systems over frequency selective fading channels," in Proc. VTC '97, Phoenix, AZ, pp. 2094-2098.

[12] A. Garcia Armada and M. Calvo, "Phase noise and sub-carrier spacing effects on the performance of an OFDM communication system," IEEE Commun. Lett., vol. 2, pp. 11-13, Jan. 1998.

[13] S. Hara and R. Prasad, "Overview of multicarrier CDMA," IEEE Commun. Mag., vol. 35, pp. 126-133, Dec. 1997.

[14] T. Pollet, P. Spruyt, and M. Moeneclaey, "The BER performance of OFDM systems using nonsynchronized sampling," in Proc. Globecom, vol. 1, San Francisco, CA, Nov. 1994, pp. 253-257.

[15] T. Pollet and M. Moeneclaey, "Synchronizability of OFDM signals," in Proc. Globecom, vol. 3, Singapore, Nov. 1995, pp. 2054-2058.

[16] M. Speth, F. Classen, and H. Meyr, "Frame synchronization OFDM systems in frequency selective fading channels," in Proc. VTC '97, Phoenix, AZ, pp. 1807-1811.

[17] P. H. Moose, "A technique for orthogonal frequency-division multiplexing frequency offset correction," IEEE Trans. Commun., vol. 42, pp. 2908-2914, Oct. 1994.

[18] P. P. Vaidyanathan, Multirate Systems and Filter Banks. Engelwood Cliffs, NJ: Prentice-Hall, 1993.
[19] K. S. Shanmugan and A. M. Breipohl, Random Signals. New York: Wiley, 1988

[20] J. G. Proakis, Digital Communications, 3rd ed. Englewood Cliffs, NJ: Prentice-Hall, 1995.

[21] P. Ho and D. K. P. Fung, "Error performance of interleaved trellis coded PSK modulations in correlated Rayleigh fading channels," IEEE Trans. Commun., vol. 40, pp. 1800-1809, Dec. 1992.

[22] L. L. Scharf, Statistical Signal Processing: Detection, Estimation, and Time Series. Reading, MA: Addison-Wesley, 1991.

[23] O. Edfors, M. Sandell, J. J. van de Beek, S. K. Wilson, and P. O. Börjesson, "OFDM channel estimation by singular value decomposition," IEEE Trans. Commun., vol. 46, pp. 931-939, July 1998.

[24] P. Hoeher, S. Kaiser, and P. Robertson, "Pilot-symbol-aided channel estimation in time and frequency," in Proc. Sixth Communication Theory Mini-Conf. Conjunction with IEEE GLOBECOM '97, Phoenix, AZ, pp. 90-96.

[25] ETSI SMG, "Overall requirements on the radio interface(s) of the UMTS,” Tech. Rep. ETR/SMG-21.02, v.3.0.0., ETSI, Valbonne, France, 1997.

[26] W. C. Jakes, Microwave Mobile Communications. New York: IEEE, 1974.

[27] S. Selby, CRC Standard Math Tables, 18th ed. Boston, MA: CRC, 1970.

Jan-Jaap van de Beek was born in Alphen aan den Rijn, the Netherlands, in 1967. He received the M.S. degree in applied mathematics from the University of Twente, the Netherlands, in 1992, and the Ph.D. degree in signal processing from Luleå University of Technology, Luleå, Sweden, in 1998.

In 1999, he joined Nokia Telecommunications, Stockholm, Sweden, as a Senior Design Engineer. His research interests include statistical parameter estimation and wireless communication systems.

Per Ola Börjesson (S'70-M'90) was born in Karlshamn, Sweden, in 1945. He received the M.Sc. degree in electrical engineering, in 1970 and the Ph.D. degree in telecommunication theory, in 1980, both from Lund Institute of Technology (LTH), Lund, Sweden. In 1983, he received the degree of Docent in Telecommunication Theory

Since 1998, he has been a Professor of signal processing at Lund University, and between 1988 and 1998, he was a Professor of signal processing at Lulea University of Technology, Luleå, Sweden. His primary research interest is in high performance communication systems, in particular, high data rate wireless and twisted pair systems. He is presently researching signal processing techniques in communication systems that use orthogonal frequency division multiplexing (OFDM) or discrete multitone (DMT) modulation. He emphasizes the interaction between models and real systems, from the creation of application-oriented models based on systems knowledge to the implementation and evaluation of algorithms.

Marie-Laure Boucheret (M'95) received the Eng. degree in electrical engineering from the Ecole Superieure des Communications de Bretagne, Brest, France, in 1985, and the Ph.D. degree in electrical engineering from the Ecole Nationale Superieure des Telecommunications, Paris, France, in 1997.

She joined ALCATEL SPACE, Toulouse, France, in 1986. She was first involved in the design of the TELECOM II $\mathrm{x}$-band transponder, and then she was engaged in the study and development of on-board digital multicarrier demodulators. She moved to the Ecole Nationale Superieure des Telecommunications, Toulouse, France, as an Associate Professor, in 1991. Her research interests include digital signal processing for communications with special emphasis on on-board processing (digital demultiplexers and beam forming networks), digital receivers (algorithms and architecture), and satellite communication systems. 
Daniel Landström was born in Göteborg, Sweden in 1972. He received the M.Sc. degree in electrical engineering from Luleå University of Technology, Luleå, Sweden, in 1996 and the Lic. Eng. degree in signal processing from Lund University, Lund, Sweden, in 1999, where he is currently working toward the Ph.D. degree in signal processing.

His research interests include statistical signal processing and parameter estimation in multicarrier communication systems. Current focus is on synchronization aspects in orthogonal frequency division mutliplexing (OFDM) systems.

Julia Martinez Arenas was born in La Roda, Spain. She received the M.Sc. degree in signal processing from the University Politecnica de Valencia, Spain, in 1994 and the Ph.D. degree in electrical engineering from the Ecole Nationale Superieure de Telecommunications in Toulouse, France, in 1998.

From 1994 to 1995, she worked as a Young Graduate Trainee at the Digital Communication Division in the European Space Research and Technology Centre in Noordwijk, the Netherlands. In 1998, she joined Ericsson Research, where she is currently working on radio access technologies and signal processing. Her reserach interests include multicarrier communication systems and evolution of GSM systems.

Per Ödling (S'90-A'95) received the M.S.E.E. degree in 1989, the licentiate degree in 1993, and the Ph.D. degree in signal processing in 1995, all from Luleå University of Technology, Luleå, Sweden.

Currently he is an Assistant Professor at the Department of Applied Electronics, Lund Institute of Technology (LTH), Lund, Sweden. Having researched cooperatively with industry over the past ten years, he is currently developing broadband internet communication over radio and twisted copper pairs. His recent work includes contributions to the standardization of the universal mobile telecommunications system (UMTS) and the very high bit rate digital subscriber loop (VDSL) within the European Telecommunications Standards Institute (ETSI), the American National Standards Institute (ANSI), and the International Telecommunication Union (ITU).
Christer Östberg was born in Malmö, Sweden, in 1959. He received the M.Sc. degree in electrical engineering in 1988 from Lund Institute of Technology (LTH), Lund, Sweden.

From 1988 to 1997, he worked at the research department for Telia in Luleå, Sweden. The main part of the R\&D topics was focused on the physical layer of multiaccess technologies for mobile communication systems. In 1997, he joined Ericsson Mobile Communication in Lund, Sweden. Presently he works as a Technical Manager with R\&D of base band algorithms for wide band coded divided multiple access (WCDMA) terminals.

Mattias Wahlqvist was born in Uppsala, Sweden, in 1969. He received the M.Sc. degree in electrical engineering in 1995 and the Lic. Eng. degree in signal processing in 1998, both from Luleå University of Technology, Sweden.

Since 1995, he has been employed by Telia Research AB and worked with the radio interface for third generation mobile communication systems. In 1998, he joined Ericsson Research, where he is currently working on radio network algorithms research. His research interests include multicarrier communication systems and algorithms for CDMA radio networks.

Sarah Kate Wilson received the A.B. degree in mathematics from Bryn Mawr College, Philadelphea, PA, in 1979, and the M.S. and Ph.D. degrees in electrical engineering from Stanford University, Stanford, CA, in 1987 and 1994, respectively.

She has worked in industry as a Programmer/Analyst and as a Research Engineer. From 1994 to 1997, she was an Assistant Professor of Electrical and Computer Engineering at Purdue University, West Lafayette, IN. She is now on the staff of the division of Signal Processing at Luleå University of Technology, Luleå, Sweden, where she is an Assistant Professor. 